\title{
Engineering complex systems
}

\section{The emergent properties of complex systems are far removed from the traditional preoccupation of engineers with design and purpose.}

\section{J. M. Ottino}

$\mathrm{C}$ omplex systems can be identified by what they do (display organization without a central organizing authority - emergence), and also by how they may or may not be analysed (as decomposing the system and analysing sub-parts do not necessarily give a clue as to the behaviour of the whole). Systems that fall within the scope of complex systems include metabolic pathways, ecosystems, the web, the US power grid and the propagation of HIV infections.

Complex systems have captured the attention of physicists, biologists, ecologists, economists and social scientists. Ideas about complex systems are making inroads in anthropology, political science and finance. Many examples of complex networks that have greatly impacted our lives - such as highways, electrification and the Internet derive from engineering. But although engineers may have developed the components, they did not plan their connection.

The hallmarks of complex systems are adaptation, self-organization and emergence - no one designed the web or the metabolic processes within a cell. And this is where the conceptual conflict with engineering arises. Engineering is not about letting systems be. Engineering is about making things happen, about convergence, optimum design and consistency of operation. Engineering is about assembling pieces that work in specific ways - that is, designing complicated systems.

It should be stressed that 'complex' is different from 'complicated'. The most elaborate mechanical watches are appropriately called très compliqué, for example the Star Caliber Patek Phillipe has over 1,000 parts. The pieces in complicated systems can be well understood in isolation, and the whole can be reassembled from its parts. The components work in unison to accomplish a function. One key defect can bring the entire system to a halt; complicated systems do not adapt. Redundancy needs to be built in when system failure is not an option.

How can engineers, who have developed many of the most important complex systems, stay connected with their subsequent development? Complexity and engineering seem at odds - complex systems are about adaptation, whereas engineering is about purpose. However, it is robustness and failure where both camps merge.

Consider the recent debate of the balance between performance and risk. Many systems

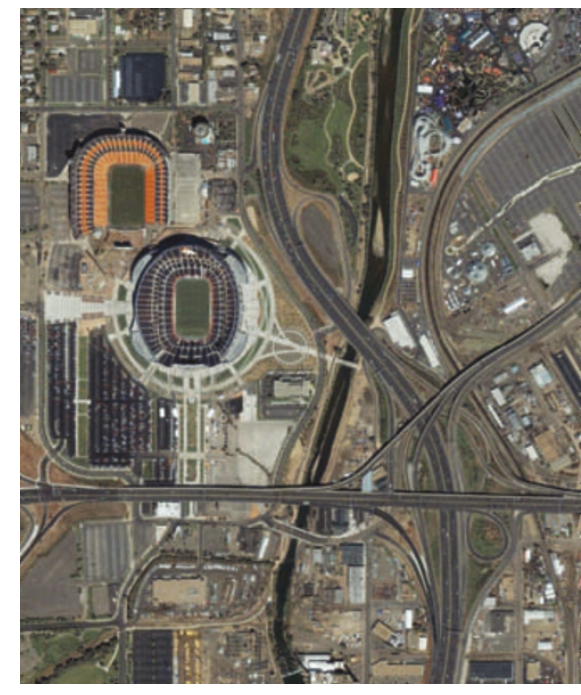

More than the sum of its parts: complex systems, such as highways, are constantly evolving.

self-organize to operate in a state of optimum performance, in the face of effects that may potentially destroy it. However, the optimal state is a high-risk state - good returns at the price of possible ruin. Most engineers are risk averse, and would prefer to eliminate the probability of catastrophic events. Recent work borrows concepts from economic theories (risk aversion, subjective benefit of outcomes) and argues that one can completely remove the likelihood of total ruin with minor loss of performance. This falls squarely in the realm of engineering, but the discussion has been driven by physics.

Engineers might also learn from social scientists. In social sciences, there is no such luxury as starting de novo - systems are already formed, one has to interpret and explain. Many engineering systems, such as the web or the US power grid, also fall into this category. How will they behave? How robust are they? How might they fail?

Although systems where self-organization has already happened present challenges, there are also opportunities in situations where self-organization can be part of the design. Could we intelligently guide systems that want to design themselves? Is it possible to actually design systems that design themselves in an intelligent manner? Self-organization and emergence have been part of materials science and engineering for quite some time, after all, lasers and superconductivity depend on collective phenomena. Emergent properties should strike a chord in materials processing, and also in the nanoworld. At larger scales, there is already work in directed self-assembly and complex dissipative systems, which organize when there is energy input. However, practical processing by self-assembly is still not a reality, and there is work here for engineers.

But the choice need not be just between designing everything at the outset and letting systems design themselves. Most design processes are far from linear, with multiple decision points and ideas 'evolving' before the final design 'emerges'. However, once finished, the design itself does not adapt. Here, engineers are beginning to get insight from biology. The emergence of function - the ability of a system to perform a task — can be guided by its environment, without imposing a rigid blueprint. For example, just like the beaks of Darwin's finches, a finite-elementanalysis of a component shape such as an airfoil can evolve plastically through a continuum of possibilities under a set of constraints, so as to optimize the shape for a given function.

Engineers calculate, and calculation requires a theory, or at least an organized framework. Could there be laws governing complex systems? If by 'laws' one means something from which consequences can be derived - as in physics - then the answer maybe no. But how about a notch below, such as discovering relationships with caveats, as in the ideal gas 'law', or uncovering power-law relationships? Then the answer is clearly yes.

Advances will require the right kinds of tools coupled with the right kind of intuition. However, the current engineering courses do not teach about self-organization, and few cover computer modelling experiments.

Despite significant recent advances in our understanding of complex systems, the field is still in flux, and there is still is a lack of consensus as to where the centre is - for some, it is exclusively cellular automata; for others it is networks. However, the landscape is bubbling with activity, and now is the time to get involved. Engineering should be at the centre of these developments, and contribute to the development of new theory and tools. J. M. Ottino is at the R. R. McCormick School of Engineering and Applied Sciences, Northwestern University, Evanston, Illinois 60208, USA.

\section{FURTHER READING}

Ball, P. Critical Mass (Heinemann, Portsmouth, 2004). Barabási, A.-L. Linked: The New Science of Networks (Perseus Publishing, Cambridge, 2002).

Hartwell, L. H. et al. Nature 402, (suppl.) C47-C52 (1999). Center for Connected Learning and Computer-Based

Modeling

http://ccl.northwestern.edu/netlogo 